Article

\title{
A Low-Power Single-Bit Continuous-Time $\Delta \Sigma$ Converter with 92.5 dB Dynamic Range for Biomedical Applications
}

\author{
Sakkarapani Balagopal * and Vishal Saxena \\ Department of Electrical and Computer Engineering, Boise State University, Boise, ID 83725, USA; \\ E-Mail: vishalsaxena@boisestate.edu \\ * Author to whom correspondence should be addressed; \\ E-Mail: sakkarapanibalagopal@u.boisestate.edu; Tel.: +1-208-596-5673; Fax: +1-208-426-2470.
}

Received: 5 April 2012; in revised form: 6 July 2012 / Accepted: 9 July 2012 /

Published: 26 July 2012

\begin{abstract}
A third-order single-bit CT- $\Delta \Sigma$ modulator for generic biomedical applications is implemented in a $0.15 \mu \mathrm{m}$ FDSOI CMOS process. The overall power efficiency is attained by employing a single-bit $\Delta \Sigma$ and a subthreshold FDSOI process. The loop-filter coefficients are determined using a systematic design centering approach by accounting for the integrator non-idealities. The single-bit CT- $\Delta \Sigma$ modulator consumes $110 \mu \mathrm{W}$ power from a $1.5 \mathrm{~V}$ power supply when clocked at $6.144 \mathrm{MHz}$. The simulation results for the modulator exhibit a dynamic range of $94.4 \mathrm{~dB}$ and peak SNDR of $92.4 \mathrm{~dB}$ for $6 \mathrm{kHz}$ signal bandwidth. The figure of merit (FoM) for the third-order, single-bit CT- $\Delta \Sigma$ modulator is $0.271 \mathrm{pJ} /$ level.
\end{abstract}

Keywords: analog-to-digital converter (ADC); continuous-time delta-sigma; data converter; oversampling; delta-sigma modulation; low-pass filter; low-power design; low-voltage design

\section{Introduction}

In recent years, there has been an increasing interest in the application of portable battery powered biomedical devices in consumer products. To increase the operation time of the battery employed in biomedical products, the system needs to be designed for low-voltage and low-power consumption. 
Several biomedical applications like electro-cardiogram (ECG), electroencephalogram (EEG), and evoked potential (EP) employ digital signal processing (DSP) techniques after digitizing the sensed analog signals. The analog-to-digital data converter (ADC), used for digitizing the sensed analog signal, is a critical and power hungry block in a generic biomedical system [1].

The ADC employed in biomedical signal processing chain typically requires $1-6 \mathrm{kHz}$ signal bandwidth with up to 14-bits or higher resolution [1]. Delta-sigma modulation based ADCs employing an oversampling technique are a well-known candidate for achieving higher resolution analog-to-digital conversion using a low-resolution quantizer [2-5]. Using oversampling techniques, the quantization noise introduced by the low-resolution quantizer is pushed outside the signal bandwidth, which results in higher resolution (SNR) and dynamic range (DR). Also, $\Delta \Sigma$ ADCs are well-suited for digitizing biomedical signals without requiring any instrumentation amplifier. When compared to other conventional ADCs, small differential-mode signal can be recovered with high resolution using a $\Delta \Sigma$ modulator [2-5].

The $\Delta \Sigma$ ADC is also proven to be well suitable for low-bandwidth signal conversion by employing oversampling, and is robust to device mismatches in the forward signal path when using nano-CMOS processes [5-7]. Traditionally, discrete-time (DT) $\Delta \Sigma$ ADC were the commonly used $\Delta \Sigma$ architecture. Many such modulators have been reported over previous decades [8,9]. However, in recent years, the continuous-time delta-sigma (CT- $\Delta \Sigma$ ) ADC is being preferred in low-power signal-processing applications due to several attractive features like an inherent anti-aliasing filter (AAF), relaxed bandwidth requirements of the active elements and lower power consumption when compared to their discrete-time counterparts $[3,5,7]$.

To enhance the power reduction in battery-powered, low-bandwidth biomedical applications, a single-bit CT $-\Delta \Sigma$ ADC is an appropriate choice. Advantages of using a single-bit quantizer include reduced chip area and significant power reduction of the $\Delta \Sigma$ loop. However, some important concerns in designing single-bit CT- $\Delta \Sigma$ include the higher in-band quantization noise due to the use of a single-bit quantizer, increased sensitivity to clock jitter and the slew rate requirement for the first operational amplifier (opamp). The higher in-band quantization noise can be addressed by increasing the effective oversampling ratio (OSR). Since the signal bandwidth requirement of battery powered biomedical applications is low, even with lower sampling rate a higher OSR can be employed. Also, due to the low sampling frequencies used in the CT $-\Delta \Sigma$ ADCs for biomedical applications, clock jitter is not a serious concern.

Any amount of slewing in the opamp introduces distortion and increases the in-band noise, resulting in a reduction of signal to quantization noise (SQNR) [10]. This should be taken care of by careful design of the opamp. The main bottleneck in achieving low-power CT- $\Delta \Sigma$ ADC design is the power requirement of the loop-filter integrators. Any power reduction in opamp reduces the finite DC gain and unity-gain frequency $\left(f_{u n}\right)$ of the opamp. The lower $f_{u n}$ of opamp impairs the characteristics of the loop-filter and thus modifies the modulator noise transfer function (NTF) with significant performance degradation. In order to achieve a low-power design with acceptable performance, the systematic design centering method proposed in [11] is adopted in this work. Further, the use of a subthreshold optimized FDSOI CMOS process in this design resulted in a low-power circuit-level implementation. 
This invited paper presents an expanded and detailed discussion on our previous work reported in [5]. The remainder of this paper is organized as follows. Section 2 illustrates the modulator architecture for this application. Section 3 demonstrates the design of the third-order modulator using systematic design centering to overcome the opamp non-idealities. Section 4 briefly explains the design of the CT- $\Delta \Sigma$ blocks. Section 5 discusses the simulation results of the designed third-order low power $\mathrm{CT}-\Delta \Sigma$. Finally, Section 6 draws conclusions about the work.

\section{Choice of Architecture}

Figure 1 shows the generalized single-ended equivalent block diagram of a single-loop CT- $\Delta \Sigma$ modulator. The $\Delta \Sigma$ ADC consists of an analog modulator followed by a digital decimation filter. In this figure, $L(s)$ is the continuous-time loop filter whose output is sampled and quantized at using a quantizer operating at a sampling rate, $f_{\mathrm{s}}$, equivalent time period $\left(T_{\mathrm{s}}\right)$. The quantizer can either be a single-bit (a comparator) or a multi-bit implementation using a Flash ADC. The modulator employs an oversampling ratio denoted by OSR and the quantizer has an excess-delay of $T_{\mathrm{d}}$, which is modeled as $\mathrm{e}^{-\mathrm{s} T \mathrm{~d}}$ in Figure 1. Here, $u(t)$ is the modulator input signal, $\mathrm{y}_{\mathrm{c}}(t)$ and $\mathrm{y}[n]$ are the continuous-time and sampled loop-filter outputs respectively, $\mathrm{v}[n]$ is the digital output of the modulator, $D_{\text {out }}[n]$ is the decimated digital output of the ADC with a clock-rate of $f_{\mathrm{s}} / \mathrm{OSR}$. In this section, we describe the various architectural choices made in our CT- $\Delta \Sigma$ modulator design.

Figure 1. A generalized single-ended block diagram of a Continuous-time $\Delta \Sigma$ Modulator.

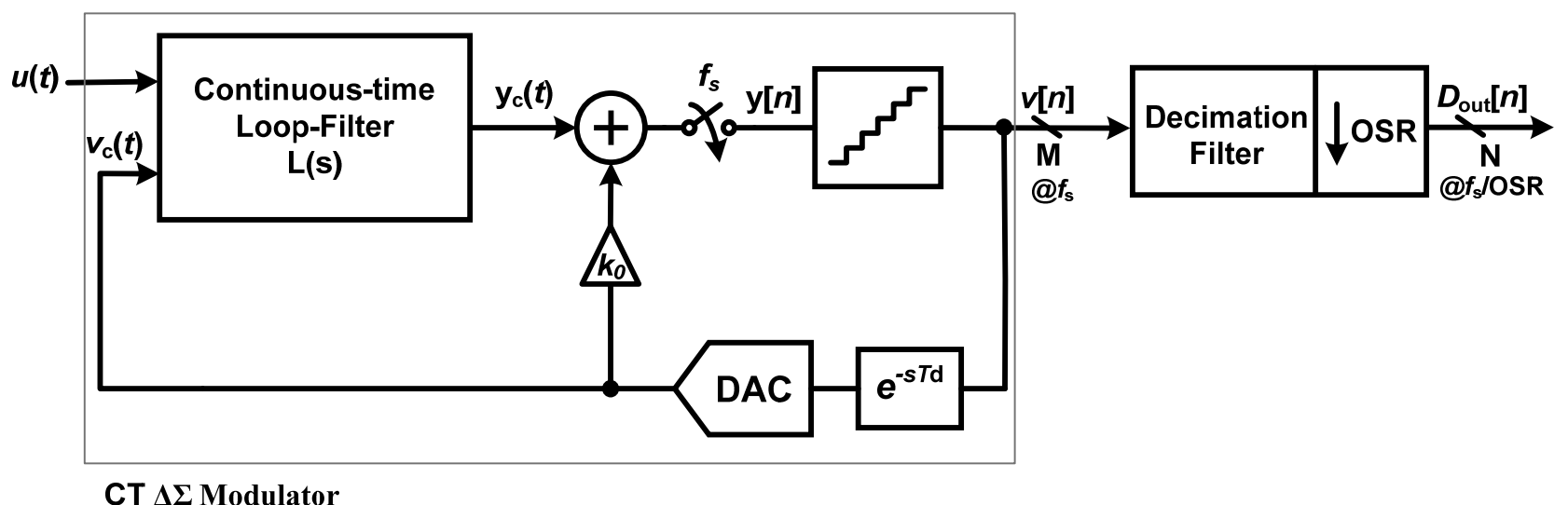

\subsection{Choice of Noise-Transfer Function NTF(z)}

The choice of proper noise transfer function, $\operatorname{NTF}(z)$, plays a prominent role in the modulator performance. In a single-bit CT- $\Delta \Sigma$ modulator design, increasing the order of the NTF may lead to instability and also reduce the maximum stable amplitude (MSA) for the input signal, which degrades the dynamic range of the ADC [1,4,5]. Thus, to achieve a SNR higher than $90 \mathrm{~dB}$ for the desired signal bandwidth with reasonable layout area, a third-order NTF with oversampling ratio of 512 is chosen. As it will be evident later, the large OSR value was chosen to avoid excessively large layout areas for the resistances and capacitances in the loop-filter. Further, for better stability of the third-order single-bit single-loop modulator, the out-of-band gain (OBG) is limited to 1.5 [12]. Also, complex zeros are placed in the signal band to improve the noise shaping performance [1,4,5]. For a stable single-bit 
CT- $\Delta \Sigma$ modulator design, it is essential to account for the effective quantizer gain $\left(k_{q}\right)$ as depicted in Figure 2. The effective gain of the single-bit quantizer is estimated by curve fitting the behavioral simulation results and is given by $k_{q}=\frac{E[v \cdot y]}{E\left[y^{2}\right]}$, where $E[x]$ is the estimation operator on the discrete-time sequence $x[n]$ [4]. Thus, an effective $N T F_{1}(z)$ is obtained by substituting a suitable value of $k_{q}$. Figure 3 shows the magnitude response of the modulator NTF fitted to the modulator's response using the effective quantizer gain $k_{q}$.

\subsection{Choice of Loop Filter Architecture}

A cascade of integrators with a distributed feedforward summation architecture is used to implement the third-order loop filter of the single-bit CT- $\Delta \Sigma$. Figure 2 shows the simple block diagram of CIFF modulator architecture.

Figure 2. Block diagram of the CIFF modulator architecture.

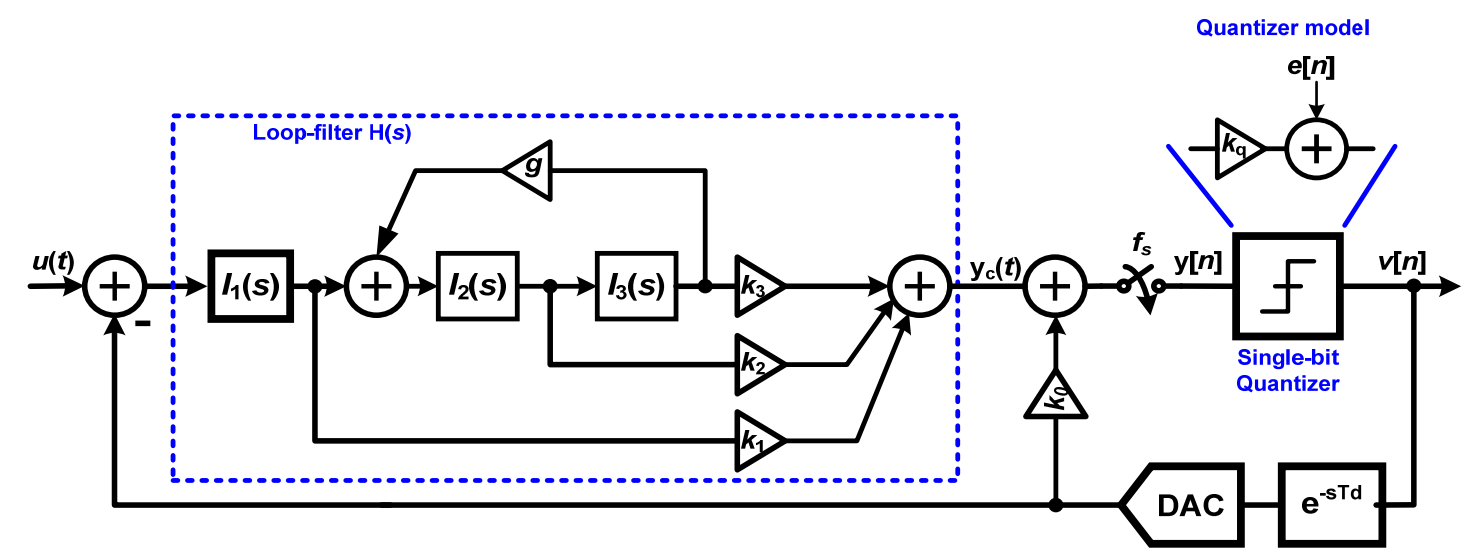

The principle advantage of feedforward architecture is that the input signal content in the successive integrator outputs is progressively scaled down from the first integrator to the last integrator in the loop-filter, and thus the loop-filter output is largely composed of the shaped quantization noise. This can be used to our benefit by scaling down the integrator performance requirements from the first to the last integrator resulting in an overall reduction in modulator power consumption. An active-RC topology is chosen for the loop filter because of its high linearity and low noise characteristics $[3,5,7,10]$. Since the first integrator sets the input referred noise and distortion for the overall modulator, the performance requirements from the first opamp are higher than the rest of the opamps [10,13]. Figure 4 shows the circuit-level schematic of the third-order CT- $\Delta \Sigma$ loop-filter along with the resistance and capacitance values used in the design. The integrators $I_{1}(\mathrm{~s}), I_{2}(\mathrm{~s})$, and $I_{3}(\mathrm{~s})$ in Figure 3 are realized using opamps $A_{1}, A_{2}$ and $A_{3}$ respectively, as shown in Figure 4. The summing node before the sampler is implemented using opamp $A_{4}$. The feedforward coefficients $\mathbf{K}\left(=\left[k_{0} k_{1} k_{2} k_{3}\right]\right)$ and summation are implemented using resistors $\left(R_{11}, R_{21}, R_{31}, R_{\mathrm{f}}\right)$ and a summing amplifier $\left(A_{4}\right)$. Since the $\mathrm{RC}$ time-constants in the loop-filter are inversely proportional to the sampling frequency $\left(f_{\mathrm{s}}\right)$, a lower sampling frequency (and thus lower OSR value) would have resulted in either very large values for resistors (1-10 $\mathrm{M} \Omega$ range) or capacitor values of $10 \mathrm{pF}$ range. Large values for resistances would result in larger layout area and higher distributed parasitic capacitance. On the other hand, large 
capacitance loads would result in higher power consumption in the opamps driving them. This provides justification for our selected OSR value of 512 .

Figure 3(a) illustrates the ideal noise shaping behavior of the CT- $\Delta \Sigma$ modulator seen in Figure 2. Here, we can see that the total noise in the signal band $(\pi / 2 \cdot \mathrm{OSR})$ is dominated by the thermal and flicker noise floor. Figure 3(b) illustrates the signal transfer function, $\operatorname{STF}(j \omega)$, of the feedforward modulator. Here, the alias suppression in the first alias-band is greater than $90 \mathrm{~dB}$, thanks to the input filtering provided by the CT loop-filter.

Figure 3. Ideal NTF and STF magnitude response of the CT- $\Delta \Sigma$ modulator.

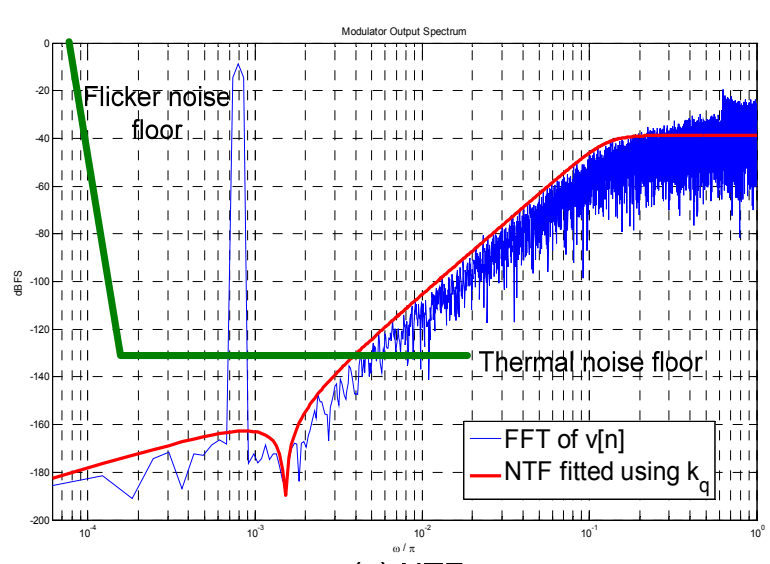

(a) NTF

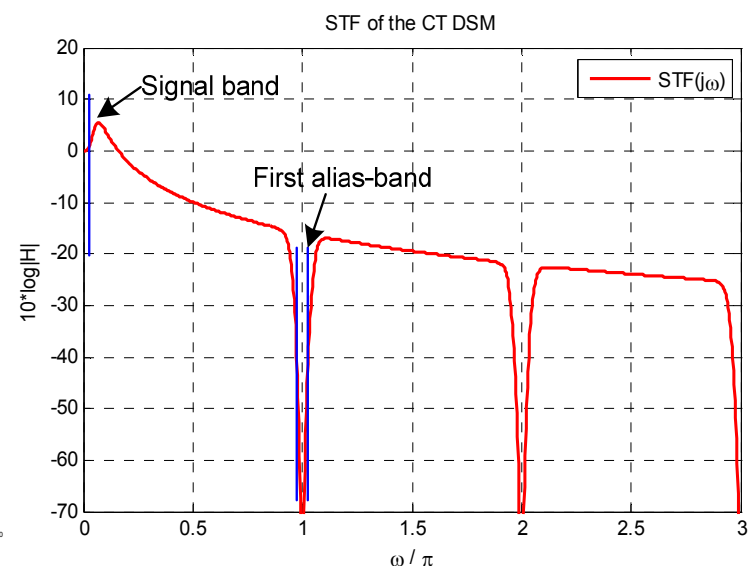

(b) STF

Figure 4. Circuit-level schematic of the feedforward loop-filter with circuit parameter values.
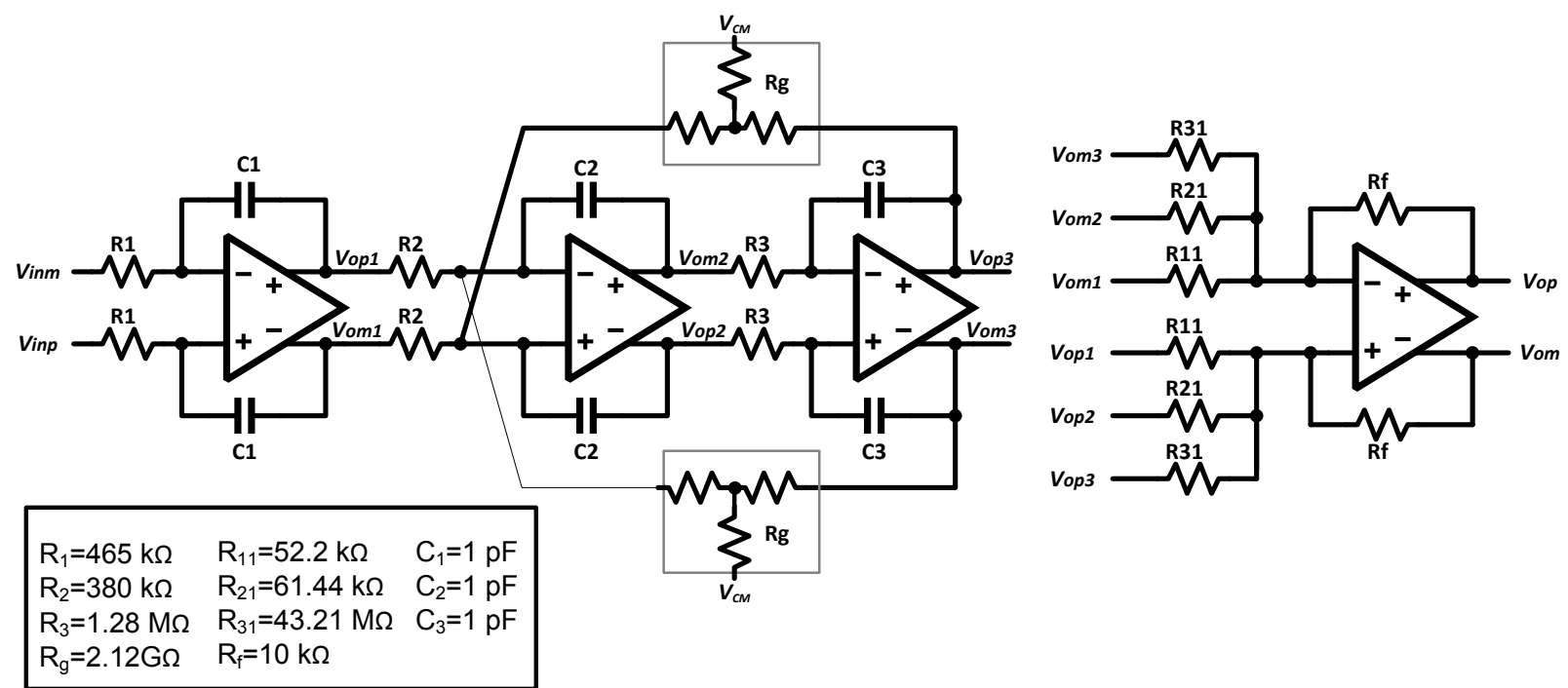

\subsection{Thermal Noise Budget}

The input referred noise of the modulator comprises of the thermal noise from the input resistor $\left(R_{1}\right)$ and the input noise of the first opamp and the feedback DAC. These noise sources dominate the overall modulator in-band noise and thus the achievable SNR. The input referred noise of the opamp is dictated by the transconductance $\left(g_{\mathrm{m} 1}\right)$ of the first stage diff-pair. In general, the values of $R_{1}$ and $g_{\mathrm{m} 1}$ 
are chosen such that each of the noise sources roughly contribute equal amount of thermal noise. Also, a PMOS differential pair with large device area is used in the first-stage of the first opamp to subside the effect of the flicker noise on the overall modulator SNR. The value of these circuit design variables $\left(R_{1}, g_{\mathrm{m} 1}\right.$, sizing of the feedback resistive DAC) have been chosen so as to keep the total thermal noise contribution to be roughly equal to $75 \%$ of the total in-band noise. This noise budgeting approach provides sufficient noise in the modulator to dither the signal input at the quantizer and help reduce spurious tones in the single-bit modulator [4].

\subsection{Clock Jitter, Excess-Loop Delay and RC Variation}

Clock jitter, excess loop delay (ELD) and RC time-constant variation are other important design concerns in a CT- $\Delta \Sigma$ [14]. However, due to low sampling frequency and negligible comparator delay of $100 \mathrm{ps}$, performance degradation due to the excess loop delay is insignificant in the present design. Also, due to the low sampling frequency required, clock jitter is not expected to degrade the modulator performance [15]. Finally, to mitigate the effect of systematic RC time-constant variation $( \pm 30 \%)$ due to process shift, a tuning method using a digitally programmable capacitor bank can be implemented [7].

\section{Loop-Filter Design Using Systematic Design Centering}

Using high-level modeling methods and tools, a loop-filter transfer function is designed using active-RC integrators. Initial modeling uses behavioral models of opamps with suitable parameters for DC gain, unity-gain bandwidth and linearity, and corresponding component values are derived for desired SNDR and dynamic range. After appropriate architecture selection of the opamps, the behavioral opamps are replaced by their transistor-level schematics.

Conventionally, the third-order loop-filter coefficients $\boldsymbol{K}=\left[k_{0} k_{1} k_{2} k_{3}\right]$, are obtained by fitting the impulse response of the discrete-time loop filter $L_{d}(z)=1-N T F^{-1}(z)$ to the continuous-time loop-filter, $L_{c}(s)$, using the impulse invariance transformation (IIT) for a given feedback DAC pulse shape [14]. The assumption in this method is that the integrators are ideal, with a transfer function given by $\frac{f_{s}}{s}$. This impulse response fitting method breaks down when applied to real integrators with finite op-amp DC gain, non-dominant opamp poles and the presence of opamp zeros. To get the desired $N T F_{\text {eff }}(z)$, either the filter coefficients $(\boldsymbol{K})$ need to be further tuned or the DC gain and the unity-gain frequency $\left(f_{u n}\right)$ of the opamp must be sufficiently large. The latter will result in an increase in the modulator power consumption [11,16]. Also, in a single-bit single-loop CT- $\Delta \Sigma$ modulator, more than $90 \%$ of the power is consumed by the opamp, which is a key design block in the loop-filter. In order to achieve low power consumption in the modulator, the bias currents in the opamps need to be optimized for the given specification. Any power reduction in the opamp impairs the characteristics of the loop-filter and thus impacts the resulting NTF performance. Figure 5 shows the magnitude response of $\operatorname{NTF}(z)$ with ideal and real integrators for the same loop-filter. It can be seen that the systematic design method restores the modulator NTF with integrators implemented using real opamps having two poles and a zero. Moreover, it is also essential that the slewing in the opamps is sufficiently limited so as not to degrade the overall modulator linearity. 
Figure 5. Magnitude responses of the resulting NTF with real integrators, with and without systematic design centering.

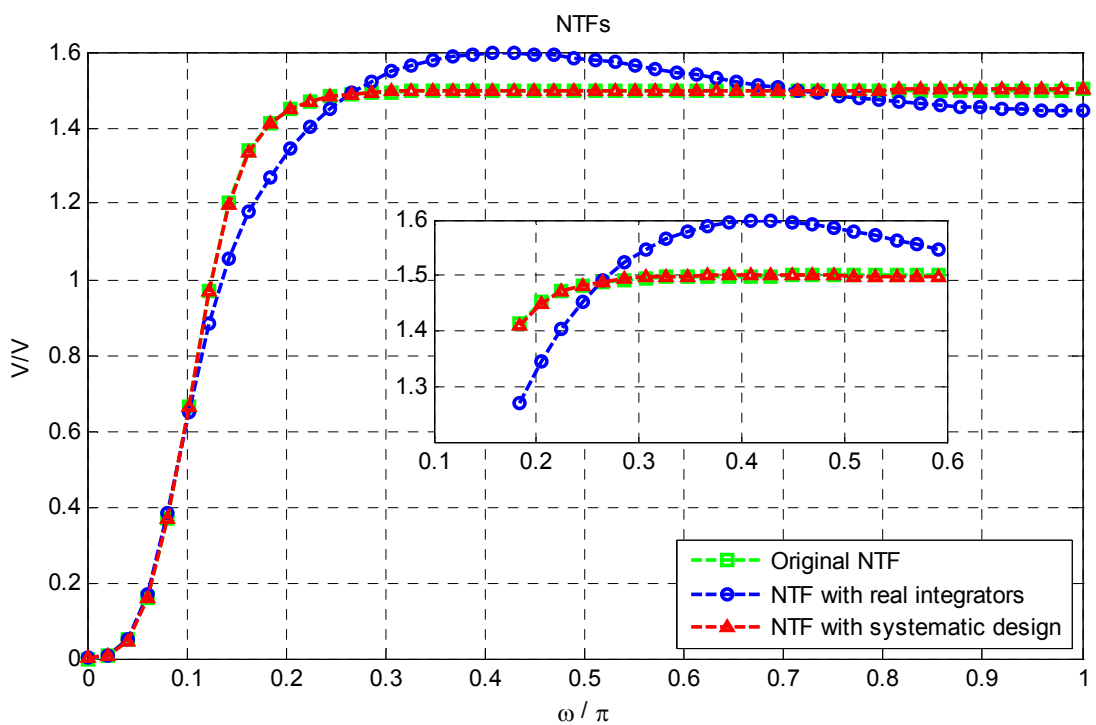

A systematic design centering method for obtaining the loop-filter coefficients by including the effect of the integrator non-idealities is employed [11]. We briefly describe this method for third-order CT- $\Delta \Sigma$ as follows: as we know, the relationship between the noise-transfer function, $\operatorname{NTF}(z)$, and the discrete-time loop-filter response, $L_{\mathrm{d}}(z)$, is given

$$
N T F_{\text {eff }}(z)=\frac{1}{1+L_{d}(z)}
$$

This relation (Equation 1) is incorporated into the impulse response fitting by using the following relation [5]

$$
\left[\begin{array}{llll}
h_{0} & h_{1} & h_{2} & h_{3}
\end{array}\right] K=\delta[n]-h[n]
$$

where $h[n]$ is the impulse response of the $\operatorname{NTF}(z)$. Further $h_{0}[n]=l_{0}[n] \otimes h[n], h_{1}[n]=l_{1}[n] \otimes h[n]$, where $l_{0}[n]$ and $l_{i}[n]$ represent the sampled DAC pulse response of direct path and at the output $i^{\text {th }}$ integrator (see [11] for details). Table 1 shows the derived loop-filter coefficients for the modulator, before and after systematic design procedure.

Table 1. Loop-filter coefficients for the modulator.

\begin{tabular}{lcccc}
\hline & $\boldsymbol{k}_{\mathbf{0}}$ & $\boldsymbol{k}_{\mathbf{1}}$ & $\boldsymbol{k}_{\mathbf{2}}$ & $\boldsymbol{k}_{\mathbf{3}}$ \\
\hline Using ideal integrators & 0 & 1.915 & 1.627 & 2.314 \\
Using real integrators & -0.038 & 0.753 & 0.239 & 0.046 \\
After systematic design with real integrators & -0.022 & 0.691 & 0.25 & 0.045 \\
\hline
\end{tabular}

As described earlier in this section, the first integrator plays a dominant role in determining the desired SNR. The first opamp $\left(A_{1}\right)$ is designed for a DC gain of $75 \mathrm{~dB}$ and a unity-gain frequency of $f_{\text {un }}=1.5 f_{s} \approx 10 \mathrm{MHz}$ with higher slewing performance $(\approx 20 \mathrm{~V} / \mu \mathrm{s})$ [3]. These values are obtained from system-level simulation in our custom developed CT- toolbox (implemented in MATLAB), using a two-pole and single-zero opamp model. Design constraints of the second and third opamp $\left(A_{2}\right.$ and $\left.A_{3}\right)$ are relaxed and are designed for $f_{\text {un }}=0.75 f_{s} \approx 4.5 \mathrm{MHz}$ to consume as low power as possible. The 
systematic design procedure assumes the adder is ideal. In reality, the adder also introduces excess delay in the loop, due to the finite $f_{\text {un }}$ of the opamp. This excess delay in turn introduces peaking in the magnitude response of the NTF and thus increasing the OBG. To mitigate this NTF peaking, $A_{4}$ is designed for $f_{\text {un }}=3 f_{s} \approx 19 \mathrm{MHz}$. The power consumption of $A_{4}$ is almost equal to that of $A_{1}$ as only $60 \mathrm{~dB}$ DC gain is used in $\mathrm{A}_{4}$.

\section{Circuit Design}

In this section, we describe the circuit level blocks in the CT- $\Delta \Sigma$ modulator. As mentioned earlier, an experimental low-power $0.15 \mu \mathrm{m}$ FDSOI CMOS process, optimized for sub-threshold (sub- $\mathrm{V}_{\mathrm{T}}$ ) operation was used for implementing the modulator. The FDSOI process provided a higher $\frac{g_{m}}{I_{D}}$ ratio for a given transistor overdrive in the sub- $\mathrm{V}_{\mathrm{T}}$ regime, and thus lead to lower-power analog circuits when compared to a bulk CMOS process. However, due to the experimental nature of the FDSOI process technology, only typical corner models were available for circuit simulation and the noise parameters were not incorporated in the BSIMSOI Spectre models.

\subsection{Opamp Design}

Figure 6 shows the schematic of the indirect compensated two-stage opamp [16]. It is established that a two-stage opamp is more efficient than the single-stage in reducing the in-band noise arising from opamp non-idealities [10]. A PMOS diff-pair is used as the first stage and pseudo class-AB buffer is used as the second stage for simple biasing and better output linearity compared to a class-A output stage [17]. $\mathrm{M}_{1}$ to $\mathrm{M}_{6}$ are long length devices to mitigate the input-referred flicker noise. Here, $C_{\mathrm{IC}}$ is the compensation capacitor and its value is chosen to be equal to $250 \mathrm{fF}$.

Figure 6. Opamp topology used in the loop-filter integrators.

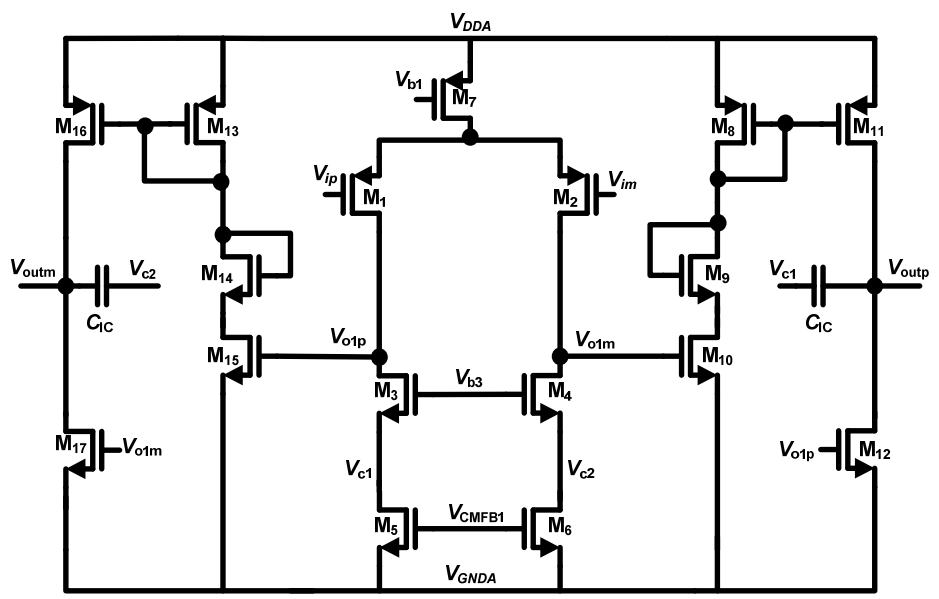

The first stage is biased to draw $5 \mu \mathrm{A}$ from the supply. To ensure the op-amp common mode output voltage is held at $V_{\mathrm{CM}}$, a common mode feedback (CMFB) loop is used in both of the opamp stages [see Figure 7(a) and (b)]. The total current drawn by the first opamp including the CMFB by the first opamp including the CMFB circuitry is $20 \mu \mathrm{A}$. The output of first stage ( $V_{\text {olp }}$ and $V_{\text {olm }}$ ) in Figure 6 is fed to the input transistors $M_{27}$ and $M_{28}$ in Figure 7(a), which averages and compares the resultant 
voltage with the $V_{\mathrm{b} 3}$ to tune the voltage $\left(V_{\mathrm{CMFB} 1}\right)$. Similarly, the CMFB circuit shown in the Figure $7(\mathrm{~b})$ adds extra current to $M_{32}$ to keep the output node ( $V_{\text {outp }}$ and $\left.V_{\text {outm }}\right)$ at $V_{\mathrm{CM}}$. This technique provides good CMFB loop stability and robustness [7]. Also, the output common-mode impedance of the opamp shown in Figure 6 is the parallel combination of positive resistance of the transistors $\left(M_{11}\right.$ and $\left.M_{16}\right)$ and the negative impedance formed by the loop $M_{10}-M_{8}-M_{11}$ and $M_{15}-M_{13}-M_{16}$. In order to avoid the negative real part in case of any mismatches in the transistors, which leads to instability of the opamp, $M_{12}$ and $M_{17}$ are made 1.5 times larger than $M_{11}$ and $M_{16}$. Hence the quiescent current in the $M_{12}$ and $M_{17}$ is 1.5 times the quiescent current in the $M_{11}$ and $M_{16}$. The remainder quiescent current for $M_{12}$ and $M_{17}$ is provided by $M_{36}$ and $M_{37}$ of the CMFB circuit as shown in the Figure 7(b).

Figure 7. CMFB circuits for the first stage (a) and second stage (b).

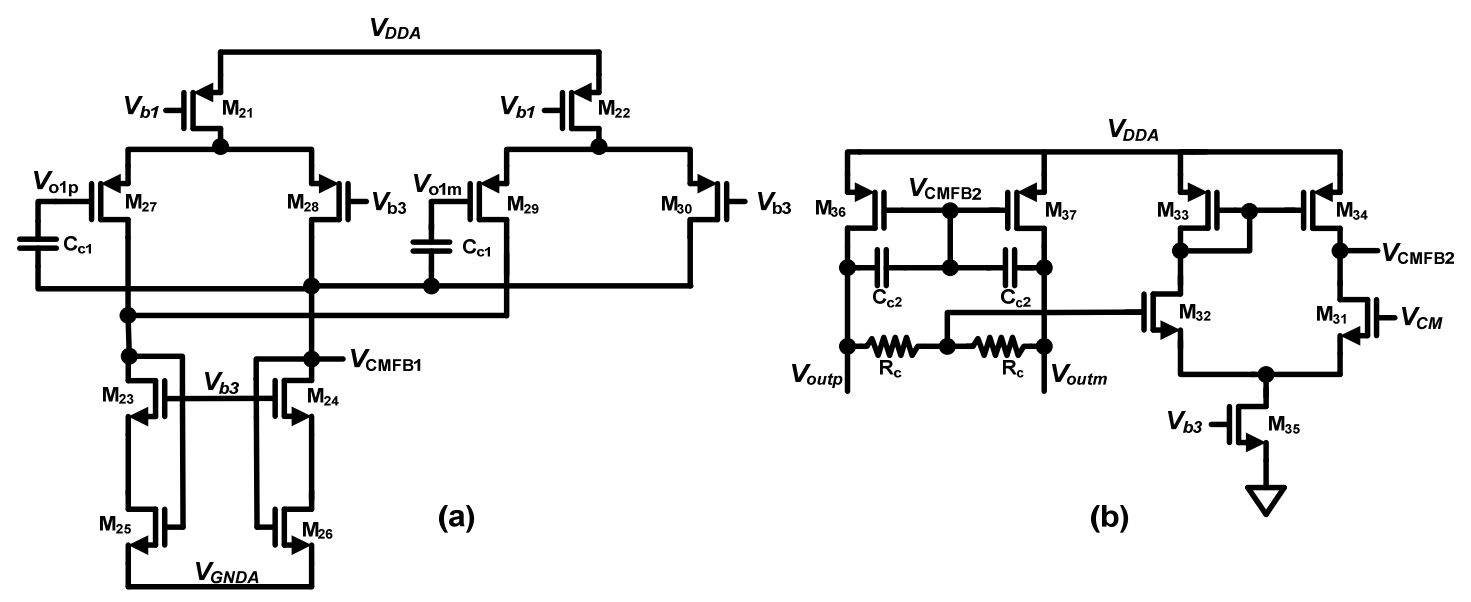

\subsection{Comparator}

Figure 8 shows the low power comparator used in the CT $-\Delta \Sigma$ modulator [4]. The comparator has a resolution of $1 \mu \mathrm{V}$ and a delay of $100 \mathrm{ps}$. The comparator is biased with a $1 \mu \mathrm{A}$ current and approximately dissipates $4 \mu \mathrm{W}$ power at a clock rate of $6.144 \mathrm{MHz}$.

Figure 8. The low-power comparator used in CT- modulator [4].

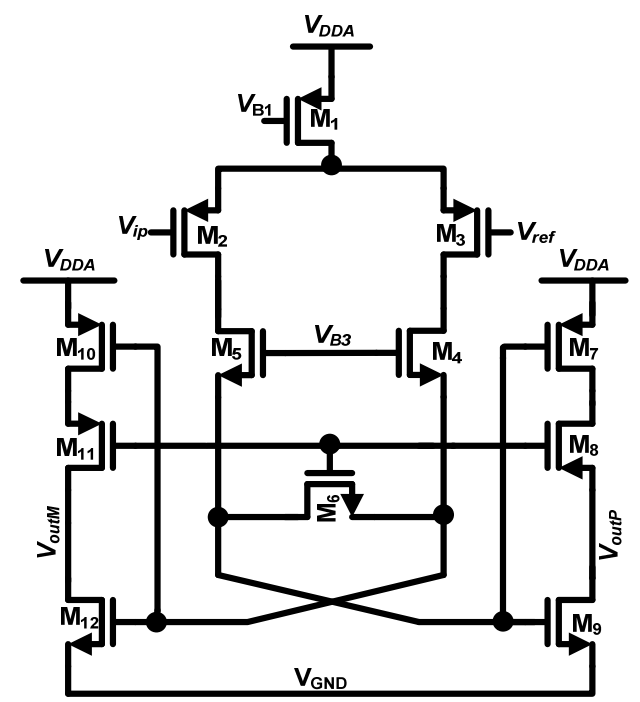




\section{Simulation Results}

The third-order CT- $\Delta \Sigma$ ADC was designed and fabricated in MIT Lincoln Lab's experimental $0.15 \mu \mathrm{m}$ FD-SOI CMOS process. Figure 9 shows the micrograph of the fabricated chip. Since the FDSOI process did not have well-controlled resistivity and capacitance, three separate ADC layouts were included on the chip. The three designs covered a spread of $50 \%$ variation in the $\mathrm{RC}$ time-constants. Sixty-four thousand output samples were collected from the modulator in the post-layout simulation. Figures 10 and 11 illustrate the post-layout simulated signal to noise and distortion ratio (SNDR). The SNDR is measured using a $2.3 \mathrm{kHz}$ input tone. The peak SNDR is close to $92.5 \mathrm{~dB}$ and the simulated dynamic range of the modulator is $94.4 \mathrm{~dB}$. Table 2 summarizes the simulated performance of the modulator. The figure of merit (FoM) of the converter is calculated using power consumption $(P)$, signal bandwidth $\left(f_{\mathrm{B}}\right)$ and SNR by using the relation [7].

$$
F O M=\frac{P}{2 \cdot f_{B} \cdot 2^{(S N R-1.76) / 6.02}}
$$

The modulator presented in this paper achieves $0.271 \mathrm{pJ} /$ level. Table 3 compares the simulated performance of the presented FDSOI design with several continuous-time $\Delta \Sigma$ ADCs from recent literature [7,18-22].

Figure 9. Fabricated chip micrograph containing the designed CT- $\Delta \Sigma$ ADCs.

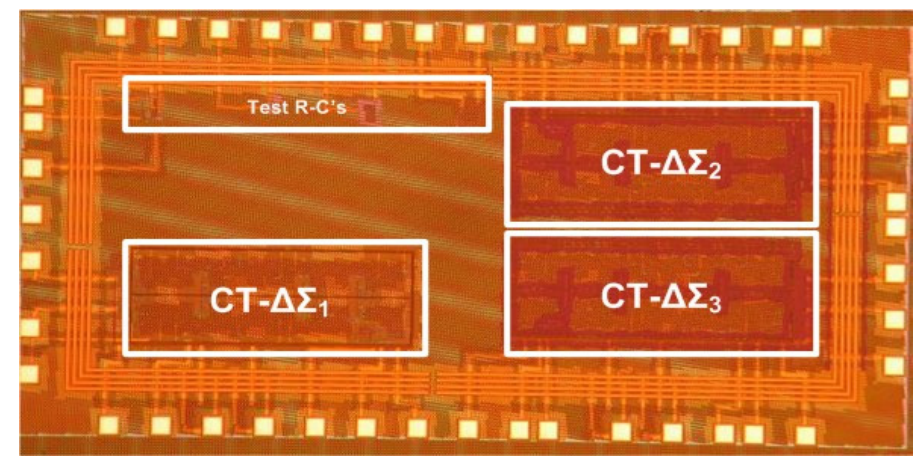

The chip was wire-bonded in a DIP package, mounted on a PCB and tested. External sinusoidal and clock inputs were applied to the chip and the output samples were collected using a mixed-signal oscilloscope. The experimental testing revealed that the value of resistances and capacitances on the chip deviated significantly from the expected values, resulting in the CT- $\Delta \Sigma$ modulators to be unstable. Our experiments led to the conclusion that at least one of the opamps in the design became unstable due to the compensation scheme breaking down with large process variation. Thus a well-characterized process with stable resistivity and capacitance parameters is necessary for such mixed-signal designs. The design presented in this paper shows significant improvement over several design except for an excellent power-optimized CT- $\Delta \Sigma$ design presented in [7]. 
Figure 10. Simulated spectrum of modulator.

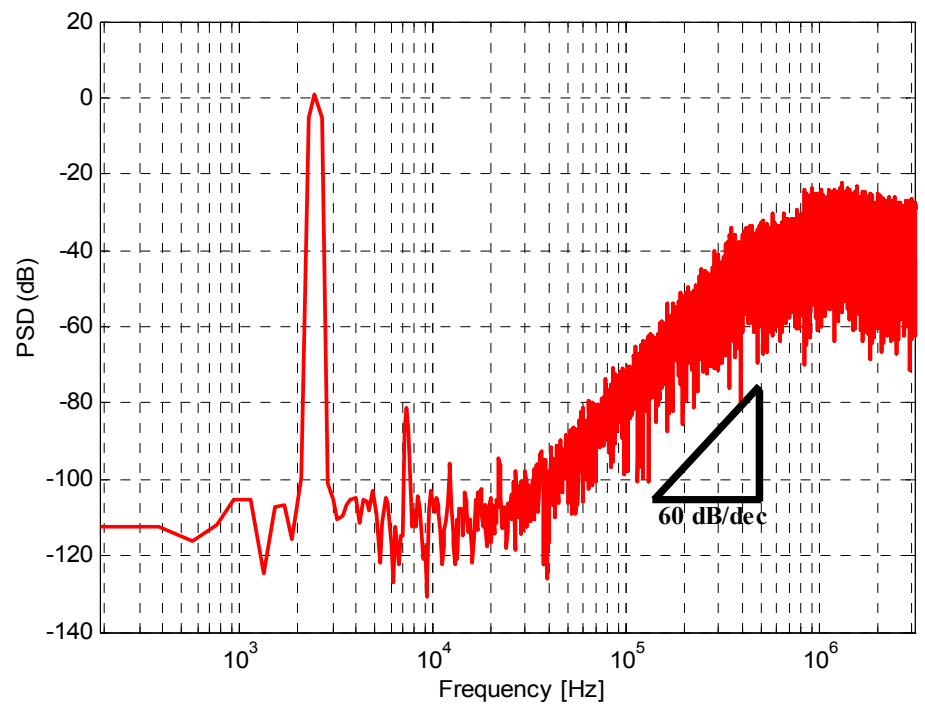

Figure 11. Simulated SNDR/Dynamic range.

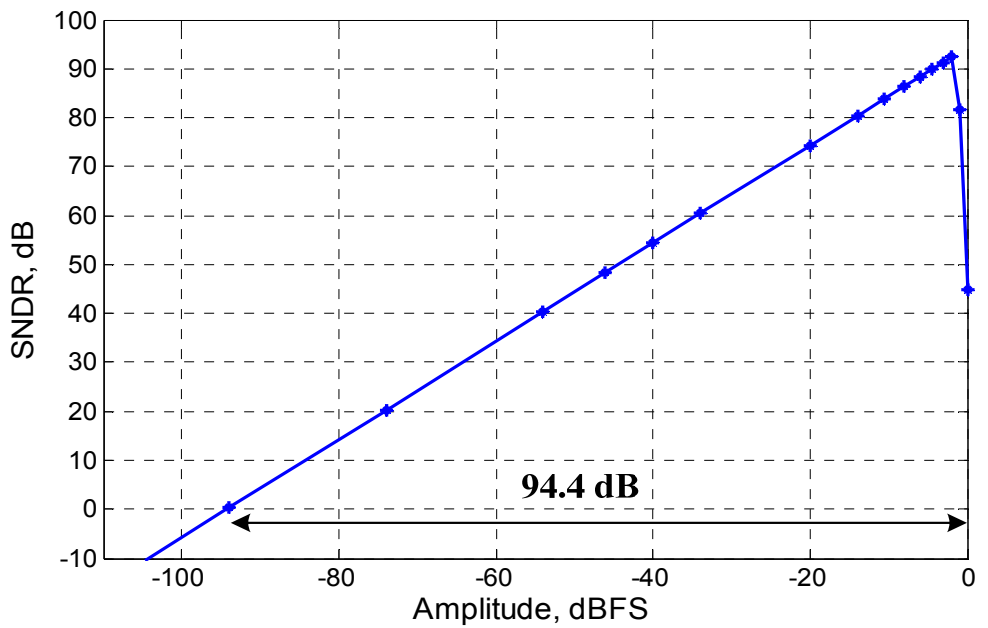

Table 2. Summary of simulated CT- $\Delta \Sigma$ modulator performance.

Signal Bandwidth/Clock Rate

Quantizer Range

Input Swing for peak SNR

Dynamic Range/ SNDR

Active Area

Process/Power Supply Voltage

Power Diss. Modulator /Reference

Figure of Merit
$6 \mathrm{kHz} / 6.144 \mathrm{MHz}$

$3 \mathrm{~V}_{\mathrm{pp}, \mathrm{diff}}$

$-1.92 \mathrm{dBFS}$

$94.4 \mathrm{~dB} / 92.4 \mathrm{~dB}$

$0.016 \mathrm{~mm}^{2}$

$0.15 \mu \mathrm{m}$ FD-SOI/1.5 V

$110 \mu \mathrm{W} / 20 \mu \mathrm{W}$

$0.271 \mathrm{pJ} /$ level 
Table 3. CT $-\Delta \Sigma$ modulator performance comparison.

\begin{tabular}{ccccccccc}
\hline Reference & Bandwidth & OSR & $\begin{array}{c}\text { Supply } \\
\text { Voltage }\end{array}$ & Technology & $\begin{array}{c}\text { Dynamic } \\
\text { Range }\end{array}$ & Power & Peak SNR & $\begin{array}{c}\text { FoM } \\
\text { (pJ/conv) }\end{array}$ \\
\hline This work & $6 \mathrm{kHz}$ & 512 & $1.5 \mathrm{~V}$ & $0.15 \mu \mathrm{m}$ FDSOI & $94.4 \mathrm{~dB}$ & $110 \mu \mathrm{W}$ & $92.4 \mathrm{~dB}$ & 0.271 \\
{$[7]$} & $24 \mathrm{kHz}$ & 64 & $1.8 \mathrm{~V}$ & $0.18 \mu \mathrm{m}$ & $93.5 \mathrm{~dB}$ & $90 \mu \mathrm{W}$ & $92.5 \mathrm{~dB}$ & 0.054 \\
{$[18]$} & $24 \mathrm{kHz}$ & 48 & $1.5 \mathrm{~V}$ & $0.35 \mu \mathrm{m}$ & $80 \mathrm{~dB}$ & $135 \mu \mathrm{W}$ & $73 \mathrm{~dB}$ & 0.74 \\
{$[19]$} & $24 \mathrm{kHz}$ & 48 & $1.5 \mathrm{~V}$ & $0.35 \mu \mathrm{m}$ & $81 \mathrm{~dB}$ & $250 \mu \mathrm{W}$ & $66 \mathrm{~dB}$ & 3.07 \\
{$[21]$} & $20 \mathrm{kHz}$ & 128 & $3.3 \mathrm{~V}$ & $0.35 \mu \mathrm{m}$ & $106 \mathrm{~dB}$ & $18 \mathrm{~mW}$ & $99 \mathrm{~dB}$ & 6.18 \\
{$[22]$} & $20 \mathrm{kHz}$ & 300 & $1.5 \mathrm{~V}$ & $65 \mathrm{~nm}$ & $95 \mathrm{~dB}$ & $2.2 \mathrm{~mW}$ & $77 \mathrm{~dB}$ & 9.51 \\
\hline
\end{tabular}

\section{Conclusions}

A low-power, single-bit CT- $\Delta \Sigma$ modulator was designed in a $0.15 \mu \mathrm{m}$ FD-SOI CMOS process for data conversion employed in generic biomedical applications. The CT loop-filter coefficients were systematically obtained by incorporating the op-amp non-idealities. This method resulted in robust modulator NTF and lower static currents in the opamp. The post-layout simulation results of the CTM exhibit a peak SNDR of $92.4 \mathrm{~dB}$, a dynamic range of $94.4 \mathrm{~dB}$ with a maximum stable input (MSA) of $-1.92 \mathrm{dBFS}$. The modulator dissipates $110 \mu \mathrm{W}$ power from a $1.5 \mathrm{~V}$ supply and achieves a FoM of $0.271 \mathrm{pJ} / \mathrm{bit}$. Future work entails fabrication of the chip in a more stable process, investigation of methods for further reducing power in the opamps in the modulator using true subthreshold design, and adaptively tuning to the mitigate the effects of $\mathrm{RC}$ time-constant variation in the CMOS process.

\section{Acknowledgments}

The fabrication of the FD-SOI test chip has been supported by DARPA and MIT Lincoln Laboratory.

\section{References}

1. Van Helleputte, N. A flexible system-on-chip (SoC) for biomedical signal acquisition. Sens. Actuators A. Phys. 2008, 142, 361-368.

2. Norsworthy, S.; Schreier, R.; Temes, G. Delta-Sigma Data Converters: Theory, Design, and Simulation; IEEE Press: New York, NY, USA, 1996.

3. Gerfers, G.; Ortmanns, M.; Manoli, Y. A 1.5-V 12-bit power-efficient continuous-time third-order $\Delta \Sigma$ modulator. IEEE J. Solid State Circuits 2003, 38, 1343-1352.

4. Schreier, R.; Temes, G. Understanding $4 \Sigma$ Data Converters; IEEE Press: Piscataway, NJ, USA, 2005.

5. Balagopal, S.; Roy, R.M.; Saxena, V. A $110 \mu \mathrm{W}$ single-bit continuous-time $\Delta \Sigma$ converter with 92.5dB dynamic range. In Proceedings of 2010 IEEE Dallas Circuits and Systems Workshop (DCAS), Richardson, TX, USA, 2010; pp. 1-4.

6. Ortmanns, M.; Manoli, Y.; Gerfers, G. A continuous-time sigma-delta modulator with reduced jitter sensitivity. In Proceedings of IEEE European Solid-State Circuits Conference, Florence, Italy, 2002. 
7. Pavan, S.; Krishnapura, N.; Pandarinathan, R.; Sankar, P. A power optimized continuous-time $\triangle \Sigma$ ADC for audio applications. IEEE J. Solid State Circuits 2008, 43, 351-360.

8. Zhiheng, C.; Tongyu, S.; Shouli, Y. A $14 \mathrm{~mW} 2.5 \mathrm{MS} / \mathrm{s} 14$ bit $\Sigma \Delta$ Modulator Using Split-Path Pseudo-Differential Amplifiers. IEEE J. Solid State Circuits 2007, 42, 2169-2179.

9. Geerts, Y.; Marques, A.M.; Steyaert, M.S.J.; Sansen, W. A 3.3 V, 15 bit, Delta-Sigma ADC with a signal bandwidth of $1.1 \mathrm{MHz}$ for ADSL applications. IEEE J. Solid State Circuits 1999, 34, 927-936.

10. Sankar, P.; Pavan, S. Analysis of integrator nonlinearity in a class of continuous-time delta-sigma modulators. IEEE Trans. Circuits Syst. II Exp. Briefs 2007, 54, 1125-1129.

11. Pavan, S. Systematic Design Centering of Continuous Time Oversampling Converters. IEEE Trans. Circuits Syst. II 2010, 57, 158-162.

12. Lee, W.L. A novel higher-order interpolative modulator topology for high resolution oversampling A/D converters. Master's thesis, Massachusetts Institute of Technology, MA, USA, June 1987.

13. Silva, J.; Moon, U.; Steensgaard, J.; Temes, G.C. Wideband low-distortion delta-sigma ADC topology. Electron. Lett. 2001, 37, 737-738.

14. Cherry, J.; Snelgrove, W. Excess loop delay in continuous-time delta-sigma modulators. IEEE Trans. Circuits Syst. II Analog. Digit. Signal Process. 1999, 46, 376-389.

15. Reddy, K.; Pavan, S. Fundamental limitations of continuous-time delta-sigma modulators due to clock jitter. IEEE Trans. Circuits Syst. I Reg. Pap. 2007, 54, 2184-2194.

16. Saxena, V.; Baker, R.J. Compensation of CMOS Op-Amps using Split-Length Transistors. In Proceedings of the 51st IEEE Midwest Symposium on Circuits and Systems, Knoxville, TN, USA, 2008, pp. 109-112.

17. Mita, R.; Palumbo, G.; Pennisi, S. Design guidelines for reversed nested Miller compensation in three-stage amplifiers. IEEE Trans. Circuits Syst. II Exp. Briefs 2003, 50, 227-233.

18. Gerfers, F.; Ortmanns, M.; Manoli, Y. A 1.5-V 12-bit power-efficient continuous-time third-order $\Sigma \Delta$ modulator. IEEE J. Solid State Circuits 2003, 38, 1343-1352.

19. Ortmanns, M.; Manoli, Y.; Gerfers, F. A continuous-time sigmadelta modulator with reduced jitter sensitivity. In Proceedings of IEEE European and Solid-State Circuits Conference, Florence, Italy, 2002; pp. 287-290.

20. Baggini, B.; Basedau, P.; Becker, R.; Bode, P.; Burdenski, R.; Esfahani, F.; Groeneweg, W.; Helfenstein, M.; Lampe, A.; Ryter, R.; Stephan, R. Baseband and audio mixed-signal front-end IC for GSM/EDGE applications. IEEE J. Solid State Circuits 2006, 41, 1364-1379.

21. Nguyen, K.; Adams, R.; Sweetland, K.; Chen, H. A 106-dB SNR hybrid oversampling analog-to-digital converter for digital audio. IEEE J. Solid State Circuits 2005, 40, 2408-2415.

22. Dorrer, L.; Kuttner, F.; Santner, A.; Kropf, C.; Hartig, T.; Torta, P.; Greco, P. A 2.2 mW, continuous-time sigma-delta ADC for voice coding with $95 \mathrm{~dB}$ dynamic range in a $65 \mathrm{~nm}$ CMOS process. In Proceedings of IEEE European and Solid-State Circuits Conference, Montreux, Switzerland, 2006; pp. 195-198.

(C) 2012 by the authors; licensee MDPI, Basel, Switzerland. This article is an open access article distributed under the terms and conditions of the Creative Commons Attribution license (http://creativecommons.org/licenses/by/3.0/). 